Dutta H

Sinha B K

Baskota D K

Ganesh Man Singh Memorial

Academy of ENT and Head \& Neck

Studies, Institute of Medicine (IOM),

Maharajgunj, Kathmandu, Nepal.

\section{Correspondence to:}

Dr. Heempali Dutta

Department of ENT-HNS

Ganesh Man Singh Bhawan,

TU Teaching Hospital

Institute of Medicine,Kathmandu,

Nepal.

e-mail:heempalidutta@hotmail.com

\title{
RECURRENT LARYNGEAL NERVE PALSY AFTER THYROID SURGERY AND LITERATURE REVIEW
}

Objective :

To observe the recurrent laryngeal nerve (RLN) status after thyroid surgery done for various types of thyroid pathology.

Material and Methods:

It was a prospective observational study done from 1st of November 2006 to 31st of October 2010 in Ganesh Man Singh Memorial Acadamy of ENT \& HN Studies, Institute of Medicine, Tribhuvan University Teaching Hospital, Maharajgung, Kathmandu. Patients undergoing different types of thyroid surgeries for both malignant and non malignant thyroid pathology were included in the study. The status of recurrent laryngeal nerve during surgery and early postoperative period were observed.

\section{Results :}

There were total 112 cases of thyroid surgery done over a period of four year. Recurrent laryngeal nerve ( RLN) palsy was observed in 15 cases in immediate postoperative period. Out of which, 10 cases fully recovered within 3 weeks of surgery and 5 cases didn't show any recovery. In 2 cases, recurrent laryngeal nerve was sacrificed because it was involved by the thyoid malignancy. The nerve was not identified in 5 cases because it was involved by the disease in 2 cases and in remaining 3 cases anatomy was distorted due to large size of thyroid mass.

\section{Conclusion:}

RLN palsy is one of the common complication after thyroid surgery. Most of the palsy are recovered within immediate postoperative periods. Meticulous surgical dissection and identification of nerve may decrease the rate of RLN palsy.

Ke y w o r d s: recurrent laryngeal nerve, thyroid, palsy.

\section{INTRODUCTION:}

Thyroid surgery is one of the common surgeries performed in head and neck region. Recurrent laryngeal nerve paralysis is one of the most frequent and serious complications after thyroid operation. Permanent nerve palsy may occur in $0 \%$ to $2.1 \%$, with an average of approximately $0.5 \%$ to $1 \%$. Temporary palsy varies from $2.9 \%$ to over $10 \%{ }^{1}$. The right-sided nerve is at higher risk due to its wide anatomical variation as compared to the left one. This complication rate can be minimized by accurate anatomical knowledge and meticulous surgical technique. The aim of this study is to assess the recurrent laryngeal nerve status during intraoperative period and early postoperative period.

\section{MATERIALS AND METHODS:}

This was a prospective observational study done from 1st of November 2006 to 31st of October 2010 in Ganesh Man Singh Memorial Acadamy of ENT \& HN Studies, Institute of Medicine, Tribhuvan University Teaching Hospital, Maharajgung, Kathmandu,Nepal. Patients undergoing thyroid surgeries for both malignant and non malignant conditions were included in the study. Patients who had preoperative recurrent laryngeal palsy were excluded from the study as well as the patients undergoing isthmusectomy and enucleation of cyst were also excluded because recurrent laryngeal were not identified and handled in these cases. All the surgeries were performed by senior surgeon. The recurrent laryngeal nerve was identified in tracheoesophageal groove and preserved. The status of nerve during intraoperative period, type of thyroid surgery were noted. The vocal cord mobility was assessed during six days of hospital stay and on 3rd week of surgery. Postoperative vocal cord palsy was defined as the presence of an immobile vocal cord or the decreased movement of the vocal cord during phonation.

\section{RESULTS:}

A total no of 112 cases of thyroid surgery were performed over a period of four years. Six cases were excluded from the study. Among excluded cases, one patient underwent enucleation of cyst, two patients had isthmusectomy where recurrent laryngeal nerve was not handled. Three patient of thyroid malignancy in whom recurrent laryngeal nerve palsy was present preoperatively were also excluded from the study.

\begin{tabular}{|c|c|}
\hline Table 1: Showing preoperative diagnosis \\
\hline Preoperative diagnosis & No of patients \\
\hline Colloid goiter & 42 \\
\hline Papillary carcinoma & 23 \\
\hline Multinodular goiter & 22 \\
\hline Follicular neoplasm & 10 \\
\hline Cystic nodule & 6 \\
\hline Hurthle cell neoplasm & 6 \\
\hline Medullary carcinoma & 3 \\
\hline Total & 112 \\
\hline
\end{tabular}

Table 1 shows the preoperative FNAC diagnosis. Colloid goiter was the most common pathology observed in thyoid gland.

\begin{tabular}{|l|l|}
\hline Table 2: Showing operative procedure. \\
\hline Operative procedure & No of patients \\
\hline Hemithyroidectomy & 65 \\
\hline Total thyroidectomy & 19 \\
\hline Subtotal thyroidectomy & 16 \\
\hline Neartotal thyroidectomy & 8 \\
\hline Completion thyroidectomy & 4 \\
\hline Total & 112 \\
\hline Table 3: Showing operative procedure and recurrent laryngea \\
\hline nerve palsy & \\
\hline Operative procedure & No of patients developing RLN palsy \\
\hline Hemithyroidectomy & 4 \\
\hline Total thyroidectomy & 6 \\
\hline Subtotal thyroidectomy & 2 \\
\hline Neartotal thyroidectomy & 1 \\
\hline Completion thyroidectomy & 2 \\
\hline Total & 15 \\
\hline
\end{tabular}

The nerve was not identified in 5 cases because it was involved by the disease in 2 cases and also because of large size of the thyroid mass which had distorted the anatomy in 3 cases. 
Table 4: Showing recovery of recurrent laryngeal nerve palsy after 3 weeks of surgery.

Fully recovered 10

Not recovered

Total no of patients

Table 4 shows the recovery of RLN palsy after 3 weeks of surgery. Ten patients had complete recovery while five patients did not show any improvement in nerve function even after 3 weeks of surgery. Among those five patients, three patients had total thyroidectomy done for papillary carcinoma (two patients) and medullary carcinoma (one patient) and two cases were of completion thyroidectomy where nerve recovery was not seen even after $3^{\text {rd }}$ week of surgery. In these cases, recurrent laryngeal nerve was not identified in three and sacrificed in two because of nerve involvement by the disease.

\section{DISCUSSION:}

Recurrent laryngeal nerve injury is a disabling complication of thyroid surgery. Because of the close anatomical relationships between thyroid gland and laryngeal nerves, impairment of the laryngeal function is a well known possible complication of thyroid surgery. Recurrent laryngeal nerve injury can lead to temporary as well as permanent palsy. RLN innervates all the intrinsic muscles of the larynx with exception of the cricothyroid muscle, which is supplied by the superior laryngeal nerve. ${ }^{2}$ Mechanism of injury to the nerve includes complete or partial transaction, traction, contusion, crushing injury, thermal damage, misplaced ligature or compromised blood supply. Any thing that increases local scar formation e.g. thyroiditis, previous surgery and radiation, increases the chances of RLN injury. 2,3 Bilateral RLN paralysis may manifest immediately after extubation and patient exhibit signs of airway obstruction in the immediate postoperative period. Bilateral RLN injury is a severe, life threatening complication that results in airway obstruction and requires immediate attention. In this condition, both vocal cords remain in a median or paramedian position. As a result, the patient exhibits inspiratory stridor, dyspnoea, tachypnoea, and nasal flaring, although the voice is near normal.4,5 Routine exposure of RLN through out its course has been shown to reduce the rate of nerve injury. ${ }^{6}$ By identifying the nerve along its course, nerve injury rate of zero has been reported in the literature even after total thyroidectomy for thyroid cancer.6,7 Conversely, when nerve is not clearly identified, the reported injury rate is three to four times higher. ${ }^{2}$ We tried to identify RLN in every cases in tracheoesophageal groove and preserve it. The nerve was not identified in five cases because it was involved by the disease in two cases and in three cases large size of the thyroid mass had distorted the anatomy. Some surgeons are of the opinion that it is too dangerous practice to dissect the nerve. 8 Whereas, some surgeon believe that it is not possible to identify the recurrent laryngeal nerve in every case. In these circumstances, technique of staying close to the thyroid capsule and division of terminal branches at capsular level is recommended. 6 It is reported that prevalence of RLN injury increases with the size of the diseased gland and extent of thyroid resection.7,9,10 In our study, three cases of total thyroidectomy and two cases of completion thyoidectomy done for thyroid malignancy didn't show any recovery at the end of 3 weeks of surgery. Careful identification and meticulous thyroid dissection is essential to prevent RLN injury. Since the nineties, the incidence of laryngeal nerve paralysis has dropped to 1 to $3 \%$. Some series even report only transient laryngeal paralysis and no permanent cases. ${ }^{13,15}$ Complication rates are affected considerably by the extent of surgical experience. ${ }^{4}$ In our study, we observed RLN palsy in two cases of completion thyroidectomy. The incidence of RLN palsy may be high in completion thyroidectomy as observed by Beahrs and Vandertoll. ${ }^{11}$ They observed the incidence of $17 \%$ of vocal fold paralysis after completion thyroidectomy in a series of 548 patients.
But the incidence is decreasing as observed by Calabro et al. ${ }^{12}$ They reported a much lower incidence, $1.5 \%$ of transient RLN paralysis after completion thyroidectomy. Postoperative recurrent nerve palsy has the potential for recovery, with a recovery rate ranging from $50 \%$ to $88 \% .14,16,18$ In our study, RLN palsy was seen in 15 out of 112 cases in immediate postoperative period. But during 3 weeks period, 10 out of 15 cases fully recovered and five cases didn't show any recovery.

\section{CONCLUSION:}

Recurrent laryngeal nerve palsy is one of the common complication after throid surgery which can be avoided by meticulous thyroid dissection and identification of RLN during surgery. Most of the nerve palsy were recovered fully during early postoperative period. Only few cases where the nerve were sacrificed or involved by the disease didn't show recovery. In our study we followed the cases for only 3 weeks but however, longer followup with more sample size may give us much better knowledge about the long term recovery of RLN palsy.

\section{REFERENCES:}

1. Bliss RD, Gauger PG, Delbridge LW. Surgeon's approach to the thyroid gland: surgical anatomy and the importance of technique. World J Surg 2000; 24: 891-897.

2. Myssiorek D. Recurrent Laryngeal nerve paralysis: anatomy and etiology. Otolaryngol Clin N Am. 2004;37(1):25-44.

3. Sittel C, Stennert E, Thumfart WF, Dapunt U, Eckel HE.Prognostic value of laryngeal electromyography in vocal fold paralysis. Arch Otolaryngol Head Neck Surg 2001;127(2):155-60. 4.Sinagra DL, Montesinos MR, Tacchi VA, Moreno JC, Falco JE, Mezzadri NA et al. Voice changes after thyroidectomy without recurrent laryngeal nerve injury. J Am Coll Surg 2004;199(4):556-60.

5. Schulte KM, Roher HD. Complications in the surgery of benign thyroid disease. Acta Chir Austriaca. 2001;33(4):164-.72.

6. Wheeler $\mathrm{MH}$. Thyroid surgery and recurrent laryngeal nerve. $\mathrm{Br} \mathrm{J}$ Surg. 1999; 86(3):291-2.

7. Hermann M, Alk G, Roka R, Glaser K, Freissmuth M.Laryngeal recurrent nerve injury in surgery for benign thyroid disease: effect of nerve dissection and impact of individual surgeon in more than 27,000 nerves at risk. Ann Surg. 2002;235(2):261-8.

8. Yalcxin B. Anatomic configurations of the recurrent laryngeal nerve and inferior thyroid artery. Surgery. 2006;139(2):181-7.

9. Chou FF, Su CY, Jeng SF, Hsu KL, Lu KY. Neurorrhaphy of the recurrent laryngeal nerve. J Am Coll Surg. 2003;197(1):52-7.

10. McHenry CR. Patient volumes and complications in thyroid surgery. Br J Surg. 2002;89(7):821-23.

11. Bearhs O. H., Vandertoll D. J. Complications of secondary thyroidectomy.Surg Gynecol Obstet, 1963, 117 : 535-9.

12. Calabro S., Auguste L. J., Attie J. N. Morbidity of completion thyroidectomy for initially mis diagnosed thyroïd carcinoma. Head Neck Surg, 1988, 10 : 235-8.

13. Wax M. K., Briant T. D. R. Completion thyroidectomy in the management of well-differentiated thyroid carcinoma.Otolaryngol. Head Neck Surg, 1992, $107:$ 63-68.

14. Lamade' W, Renz K, Willeke F, Klar E, Herfarth C. Effect of training on the incidence of nerve damage in thyroid surgery. Br J Surg. 1999;86:388-391.

15. Dejong S. A., Demeter J. G., Lawrence A. M., Paloyane.Necessity and safety of completion thyroidectomy for differentiated thyroid carcinoma. Surgery, 1992, 112:734-739.

16. Martensson $\mathrm{H}$, Terins J. Recurrent laryngeal nerve palsy in thyroid gland surgery related to operations and nerves at risk. Arch Surg. 1985;120:475-477.

17. Wagner HE, Seiler C. Recurrent laryngeal nerve palsy after thyroid gland surgery. Br J Surg. 1994;81:226-228.

18. Jatzko GR, Lisborg PH, Müller MG, Wette VM. Recurrent nerve palsy after thyroid operations: principal nerve identification and a literature review. Surgery.1994;115:139-144. 